\title{
Comparative Research on the Characteristic of Aerodynamic Drag Reducing Between the Projectile with Column and Truncated-Cone Base Cavity Shape
}

\author{
Zhihong $\mathrm{Ye}^{\mathrm{a}^{*}}$ and Haibo $\mathrm{Lu}^{\mathrm{b}}$ \\ Nanjing Artillery Academy, Nanjing, 211132, China \\ asubmission621@163.com, 'lhbboo@sohu.com
}

Keywords: Projectile with base cavity; Different base cavity shape; Aerodynamic drag reducing; CFD

\begin{abstract}
In order to discuss the effect of the base cavity shape on the projectile aerodynamic force, projectile with different base cavity shape, column and truncated-cone (dilation and shrinkage one, with different incline angle " $\alpha$ ", $15^{\circ}, 10^{\circ}, 5^{\circ}, 2^{\circ}$ ), was investigated numerically. The distribution of the flow field and the aerodynamic drag of the projectile under a supersonic flow condition were obtained. Results show that the circumfluence plays a key role to effect the aerodynamic drag force of the projectile. Compare with the traditional base cavity shape (column one), dilation truncated-cone shape, increases the aerodynamic drag of the projectile, but shrinkage truncated-cone (any incline angle) is useful to reduce the aerodynamic drag of the projectile. And there is an optimum incline angle of the shrinkage truncated-cone.
\end{abstract}

\section{Introduction}

The aerodynamic drag of a projectile could be divided into three parts, base, wave and friction drag. Under the supersonic flow condition, for a common rotary projectile, the base drag make up 30 40 percent of the total aerodynamic drag [1]. Many investigations have been justified that the base cavity configuration is an effective method to reduce the base drag of the projectile [2-6]. Upon the same condition, cause of the drag reducing of the base cavity, the range of fire for projectile with base cavity is farther than the projectile without base cavity 3 5\% [7].

In the paper, projectile with the different base cavity shape, such as column and truncated-cone, was investigated numerically. The effect of the base cavity shape on the projectile aerodynamic force was discussed.

\section{Base Cavity Shape}

As shown in Fig.1, the schematic map of the projectile with different shape base cavity is given. There are two kinds of truncated-cone base cavity, dilation (b) and shrinkage (c) one. " $\alpha$ " is the incline angle.
a. column
b. truncated-cone (dilation)
c. truncated-cone (shrinkage)

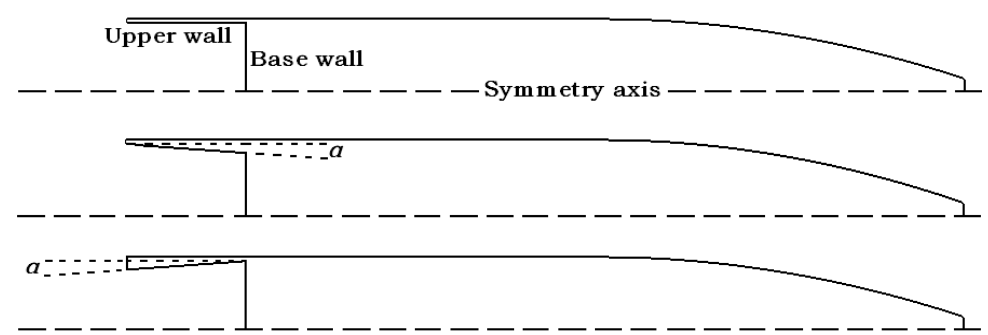

Figure 1. Schematic of the projectile shape 


\section{Numerical Method}

Governing Equation The $k-\varepsilon$ turbulence model [8] and the axisymmetric Navier-Stokes equation [9] are used in the simulation. The $\mathrm{N}-\mathrm{S}$ equation is given by

$$
\partial U / \partial T^{+} \partial F(U) / \partial x+{ }^{+} \partial G(U) / \partial r+1 / r \cdot \partial H(U) / \partial r=1 / \operatorname{Re} \cdot\left(\partial F_{v}(U) / \partial x+{ }{ }^{\partial} G_{v}(U) / \partial r+1 / r \cdot \partial H_{v}(U) / \partial r\right)
$$

where $U$ is the conservation variable, $F, G$ and $H$ are the inviscid terms, $F_{v}, G_{v}$ and $H_{v}$ are the viscous terms.

The convective terms are approximated using the Van Leer [10] splitting method and the central difference method is used for the viscous terms. The LU-SSOR scheme is used for the time integration.

Grid and Boundary Conditions As show in Table 1, the flow conditions are given. The wall boundary condition is assumed to no-slip and adiabatic.

The body-fitted grid of the projectile with base cavity (dilation truncated-cone, $\alpha=15^{\circ}$ ) is given by Fig.2.

Table 1 Boundary conditions

\begin{tabular}{|c|c|c|}
\hline Free stream parameter & Unit & Value \\
\hline Mach Number $(M a)$ & ---- & 1.97 \\
\hline Pressure $\left(p_{\infty}\right)$ & $\mathrm{Pa}$ & 101325 \\
\hline Temperature $\left(T_{\infty}\right)$ & $\mathrm{K}$ & 300 \\
\hline
\end{tabular}

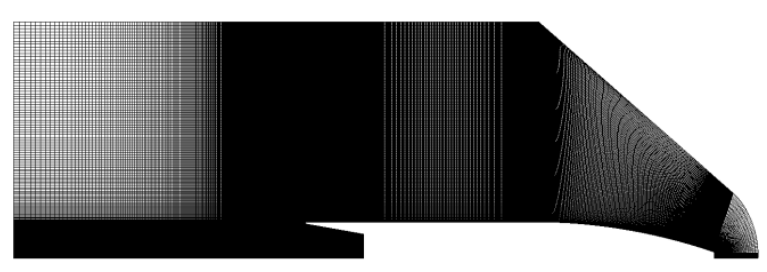

Figure 2. Grid of the simulation model (dilation truncated-cone, $\alpha=15^{\circ}$ )

\section{Results and Discussion}

Flow field The distributions of Ma contours and streamlines of the projectile with different shape base cavity are shown in Fig. 3. As show in Fig. 3, streamlines of projectile with a dilation truncated-cone base cavity are similar to the projectile with the column shape base cavity (Fig. 3 b, d, $\mathrm{f}, \mathrm{h})$. A big and fierce circumfluence located at the backside of the projectile and half of the circumfluence is inside the base cavity. But the circumfluence of the projectile with a shrinkage truncated-cone base cavity, which is limited by the boundary of the base cavity, is splitted into two parts (Fig. 3 a, c, e). With the reducing of the shrinkage truncated-cone incline angle, this effect of the splitting turns into fuzzy. As show in Fig. 3 (g), the shrinkage truncated-cone $\alpha=2^{\circ}$, the shape of the base cavity has no splitting effect on the circumfluence, and it is similar to the projectile with the column shape base cavity.

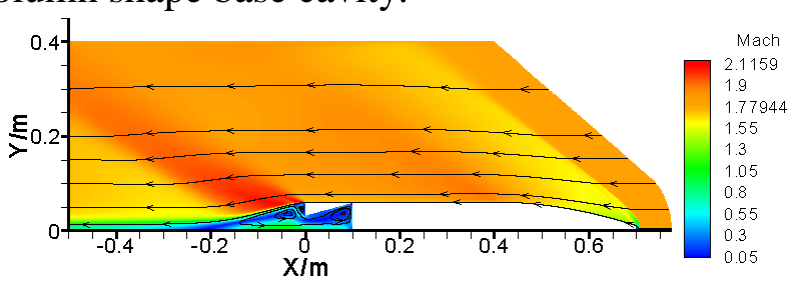

a shrinkage $\alpha=15^{\circ}$

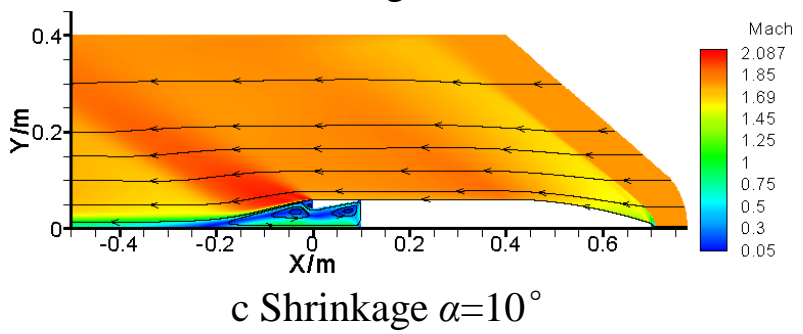

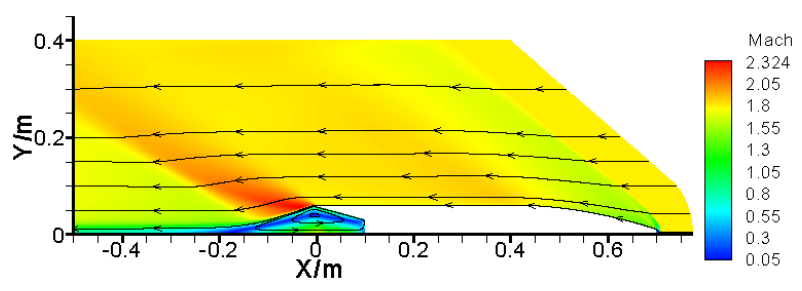

b Dilation $\alpha=15^{\circ}$

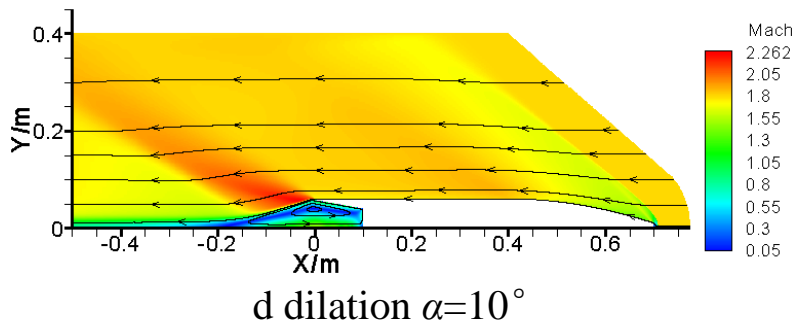




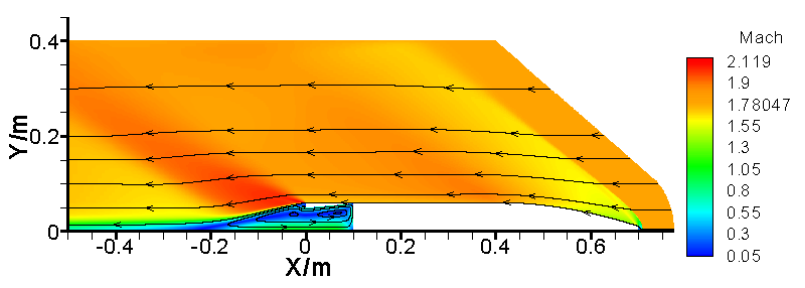

e Shrinkage $\alpha=5^{\circ}$

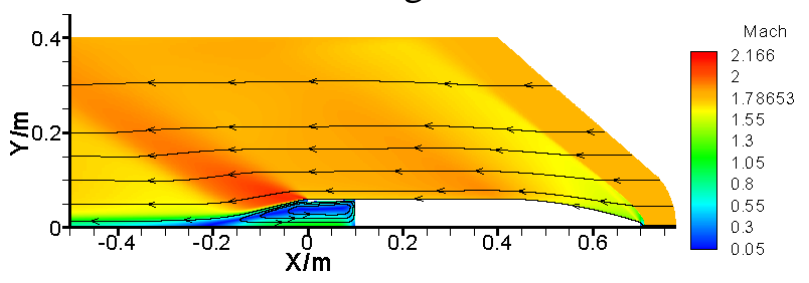

g shrinkage $\alpha=2^{\circ}$

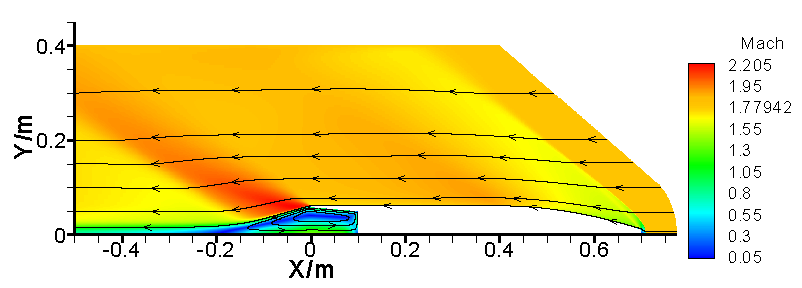

f dilation $\alpha=5^{\circ}$

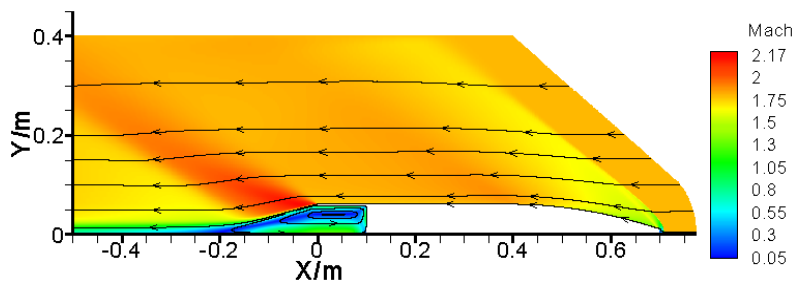

$\mathrm{h}$ column

Figure 3. Distributions of the Ma contours and the streamlines

As show in Fig. 4, the distribution of temperature (Shrinkage, $\alpha=5^{\circ}$ ) is given. High temperature area was formed behind the bottom of the cavity and inside the cavity respectively. The shape of the base cavity has little effect on the distribution of the temperature. The distribution of pressure (dilation, $\alpha=5^{\circ}$ ) is given in Fig. 5. The main difference of each projectile with different base cavity is located inside the cavity, and these difference causes to the different aerodynamic drag reducing efficiency of the base cavity.

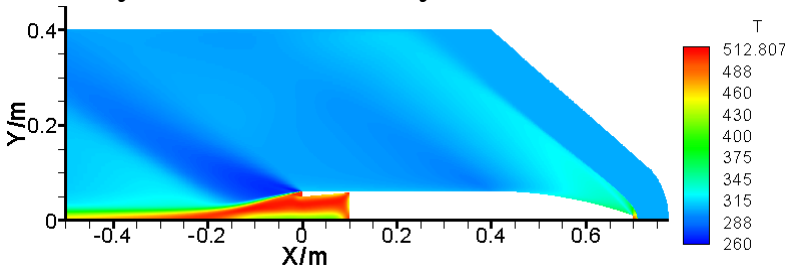

Figure 4. Distributions of the temperature (shrinkage, $\alpha=5^{\circ}$ )

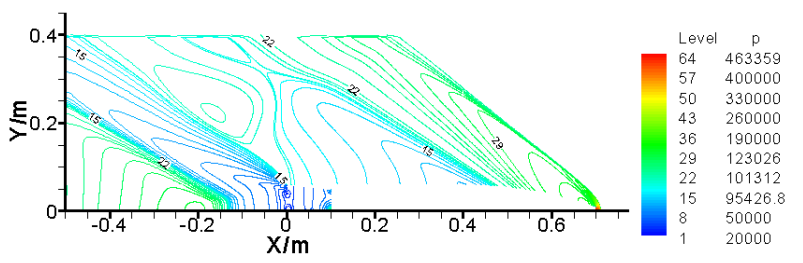

Figure 5. Distributions of the pressure (dilation, $\alpha=5^{\circ}$ )

Aerodynamic Drag As shown in Fig. 6, aerodynamic drag coefficient $\left(C_{d}\right)$ of each projectile with different shape base cavity is given. The aerodynamic drag coefficients of the projectile with dilation truncated-cone base cavity are bigger than the projectile with a column base cavity. Base cavity with a dilation truncated-cone has no help to reduce the aerodynamic drag of the projectile. The aerodynamic drag coefficients of the projectile with shrinkage truncated-cone base cavity are smaller than the projectile with a column base cavity. Base cavity with a shrinkage truncated-cone is useful to reduce the aerodynamic drag of the projectile. What's more, there is an optimization incline angle of the shrinkage truncated-cone.

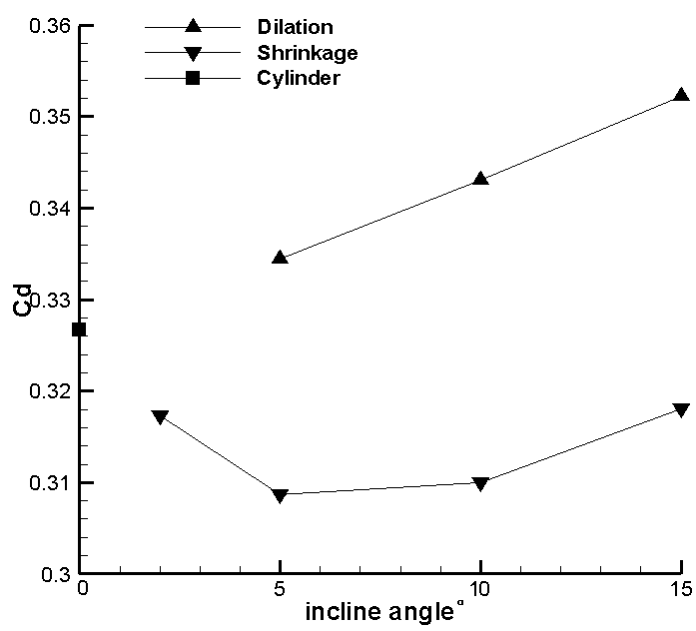

Figure 6 Distributions of the aerodynamic drag coefficient $\left(C_{d}\right)$ 


\section{Conclusion}

Projectile with the different base cavity shape, such as column and truncated-cone (dilation and shrinkage one, with different incline angle " $\alpha$ ", $15^{\circ}, 10^{\circ}, 5^{\circ}, 2^{\circ}$ ), was investigated numerically. The distribution of the flow field and the aerodynamic drag force of the projectile under a supersonic flow condition were obtained. The effect of the base cavity shape on the projectile aerodynamic force was discussed.

The shape of the base cavity has a great affect on the afterbody flow field of the projectile.

Between the afterbody flow field, the circumfluence plays a key role to effect the aerodynamic drag force of the projectile.

Compare with the traditional base cavity shape (column one), base cavity with a dilation truncated-cone shape, any incline angle, increases the aerodynamic drag of the projectile. It is no useful to reduce the aerodynamic drag of the projectile.

On the contrary, base cavity with a shrinkage truncated-cone (any incline angle) is useful to reduce the aerodynamic drag of the projectile, and there is an optimum incline angle of the shrinkage truncated-cone, which is between $2^{\circ} \sim 10^{\circ}$.

\section{References}

[1] Sheng Zhongshu, Liu Yafei. Projectile Aerodynamics. Beijing: National Defense Industry Press, 1984. (in Chinese) pp.36

[2] Cao Jiayi, Lu Chuanjing, Chen Ying, Chen Xin, Li jie. Research on the base cavity of a sub-launched projectile. Journal of Hudrodynamics, Vol. 24, Apr. 2012, pp. 244-249.

[3] A. Ibrahim, A. Filippone. Supersonic aerodynamics of a projectile with slot cavities. Aeronautical Journal, Vol. 114, Jan. 2010, pp. 15-24.

[4] Wang Zhongyuan. The effect about projectile of base cavity with side holes to flight drag under supersonic flow. ACTA aerodynamica sinica, 1997,15(4), 502-506. (in Chinese)

[5] Edgar M. G. Preliminary investigation of effectiveness of base bleed in reducing drag of blunt-base bodies in supersonic stream. NASA RM26, 1971.

[6] Elizabeth R. D. The effectiveness of base-bleed in reducing drag of boat-tailed bodies at supersonic velocities. PB157711, 1970.

[7] Wei Huizhi, Zhu Hesong. The design theory of projectile. Beijing: National Defense Industry Press, 1985. (in Chinese) pp.36

[8] Tao Wenquan. Numerical Heat Transfer, 2nd edn. Xi' an: Xi'an Jiaotong University Press, 2001 (in Chinese)

[9] Wu Xiaosong. The numerical investigation on flow field of projectile (with base bleed). Journal of ballistics, 4 (3), pp. 39-43, 1992 (in Chinese)

[10] Wang Xuede, Tan Junjie, Lin Xiaohong, Tang Zhenhua. Research on parallel numerical simulation of N-S equations based on Van Leer+AUSM scheme. Jounal of Astronautics, Vol. 31, Apr. 2010, pp. 986-992. (in Chinese) 